\title{
Association between an Incomplete Vaccination Schedule and Nosocomial Sepsis among Children with Cancer
}

\author{
Jesus Reyna-Figueroa, ${ }^{1,2}$, Diana PerezPeña-Rosas ${ }^{2}$, Patricia Galindo-Delgado ${ }^{2}$, \\ Ana Elena Limon-Rojas ${ }^{2}$, Vicente Madrid-Marina ${ }^{1}$ \\ ${ }^{1}$ Program in Public Health Sciences, National Institute of Public Health, Mexico City, Mexico; ${ }^{2}$ Central South Hospital, Petroleos \\ Mexicanos, Mexico City, Mexico. \\ Email: ${ }^{*}$ jesusreynaf@gmail.com
}

Received January $8^{\text {th }}, 2013$; revised February $8^{\text {th }}, 2013$; accepted February $15^{\text {th }}, 2013$

\begin{abstract}
Background: Patients with cancer constitute a special group where immunization programs are often interrupted to begin treatment with chemotherapy. Sepsis is one of the main complications in this group. Methods: A hospital-based case-control study matched by age was carried out among subjects $\leq 9$ years of age with cancer diagnosis. Children with cancer without sepsis and children with surgical pathology were included as controls; children with sepsis were included as cases. A bivariate logistic regression was used to determine the factors associated to nosocomial sepsis, and odds ratios were calculated with $95 \%$ confidence intervals. The percentage of attributable risk was calculated for the variables included in the final model. Results: Nineteen children with cancer and sepsis and 83 controls were included. Twelve (44\%) cases had an incomplete vaccination schedule according to their age. The association force between incomplete schedule and sepsis was 10.1 (95\% CI, 3 - 36; $p<0.05)$. Conclusions: Approximately, $20 \%$ to $65 \%$ of the cases of serious nosocomial infection can be associated to an incomplete vaccination schedule. Strategies should be implemented to improve the general pediatric population's vaccination status before a serious disease, such as cancer or another chronic condition preventing the application of vaccines, develops.
\end{abstract}

Keywords: Vaccines; Cancer; Pediatrics; Immunocompromise; Association

\section{Introduction}

A country's health programs should include sanitary policies that address both infectious and non-infectious diseases. This is normally achieved by adding actions aimed to decrease other ailments considered as public health issues to infectious diseases control strategies [1,2].

Policies comprised in the "universal vaccination schedule" are considered the most successful health intervenetions worldwide, since they have shown to be decisive elements in terms of the epidemiological change occurred in recent years. Their impact is clearly made evident in the decrease of mortality due to acute diarrheal disease after the anti-rotavirus vaccine was added, in the eradication of smallpox and in the control of poliomyelitis with the oral vaccine [3-5].

The operational population and biological argument of mass immunization is considered to be one of the most complex: Its effect reduces the numbers of basic microbial reproduction, due to the impact on the agent's transmissibility. However, if for whatever reason the immunization schedule is interrupted, the original conditions

\footnotetext{
${ }^{*}$ Corresponding author.
}

for transmission eventually arise again, and the population is thus considered to be susceptible once more. This scenario includes children with cancer who are immunologically depressed due to chemotherapy. Effects on the vaccination process entail the appearance of adverse events due to the application of the immunogen and a decreased protective response, all of which determines the interruption of the schedule and enhances the development of serious infections [6,7].

One of the main complications of children with cancer in the hospital setting is sepsis. It has been reported that death due to this cause is five times higher in this population than among hospitalized children without cancer. Although neutropenia is considered the main factor furthering serious infections, theoretically an inadequate immunological memory due to an incomplete vaccination schedule can enhance the acquisition of nosocomial infections $[8,9]$.

Sepsis is considered an important public health issue since it constitutes one of the main causes of death among critically ill patients [10]. In the United States, more than 500 thousand patients with sepsis have been reported annually. There are approximately 250 thousand deaths 
[11], and the incidence is 3.0/1000 [12]. In Mexico, it accounts for $13.8 \%$ of nosocomial infections, and the number rises to $21.6 \%$ if cases of systemic candidiasis and bacteremia are included $[13,14]$. According to the Health Ministry in Mexico, leukemia is the most common cancer among children: There are 60 to 100 new cases per 1 million inhabitants per year $(81.5 \%$ of which correspond to acute lymphoblastic leukemia), and it is the second cause of death among individuals between 1 and 14 years of age $[15,16]$ (Figure 1).

At our institution, we have recently established that $46 \%$ of children diagnosed with cancer start chemotherapeutic treatment with an incomplete vaccination schedule. We proposed to carry out an analysis of the patients in order to determine the association between the incomplete vaccination schedule and nosocomial sepsis in this group.

\section{Material and Methods}

Design: A hospital-based case-control study matched by age was carried out taking into account four controls per case. Subjects included nine-year-old or younger patients with a variety of cancer diagnoses identified by crossing the databases from the computing department and the daily health reports from the department of pediatrics (Table 1). Afterwards, the individual electronic clinical records were reviewed to obtain demographic information; clinical and laboratory data; results of blood, cerebrospinal fluid and urine cultures, as well as to establish the suspicion of sepsis during the first hospitalization (induction chemotherapy). Following the same procedure, the keys of the potentially surgical illnesses (cardiovas cular and general surgery) were crossed and thus, the se- lection of controls was carried out, according to age.

\subsection{Selection of Participants (Cases and Controls)}

The inclusion of children ( $\leq 9$ years of age) ensured they

Table 1. General characteristics of the 46 cases of children with cancer included.

\begin{tabular}{cc}
\hline Variable & $\begin{array}{c}\text { Patients with cancer } \\
n=46\end{array}$ \\
\hline Average age (SD) & $6.2(4)$ \\
Sex $n$ (\%) & \\
Feminine & $21(45.5)$ \\
Masculine & $25(54.5)$ \\
Type of cancer & \\
Leukemia & $30(65)$ \\
Wilms tumor & $6(13)$ \\
CNS & $6(13)$ \\
Osteosarcoma & $2(4)$ \\
non-Hodgkin lymphoma & $2(4)$ \\
Complete schedule & $17(36.9)$ \\
Incomplete schedule & $29(63.4)$ \\
Hospital stay/days & 27.7 \\
Confirmed sepsis & $19(41.3)$ \\
Hepatitis B vaccine applied & $7(15.2)$ \\
Pentavalent vaccine applied & $43(93.4)$ \\
Rotavirus vaccine applied & $7(15.2)$ \\
Pneumococcal vaccine applied & $4(8.6)$ \\
Influenza vaccine applied & $8(17.2)$ \\
DPT vaccine applied & $32(69.5)$ \\
Triple viral vaccine applied & $45(97.8)$ \\
\hline
\end{tabular}

CNS $=$ Central Nervous System; DPT $=$ vaccine against diphtheria, pertussis and tetanus; $\mathrm{SD}=$ Standard Deviation.

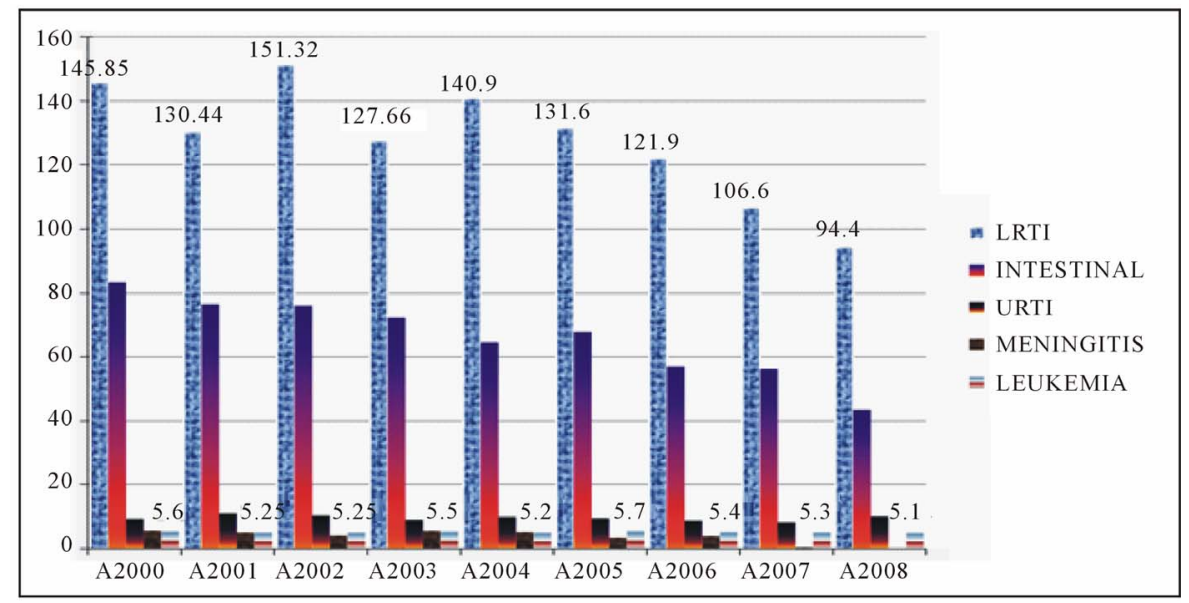

Source: Ministry of Health/General Direction of Health Information. Developed from the database on deaths 2000-2008 by INEGL/SS, the Population Projections in Mexico 2005-2050, and the retrospective projection 1990 -2004, CONAPO 2006. Rate per 100000 inhabitants from 0 to 14 years of age

LRTI = Low Respiratory Tract Infection, INTESTINAL = Intestinal Infections, URTI = Upper Respiratory Tract Infection. A = year.

Figure 1. Accumulated mortality rate in children from 0 to 14 years of age in mexico from 2000 to 2008. 
were eligible to receive the vaccines from the institutional schedule started in 2002. As from this date, hospitals have had the electronic clinical record at their disposal.

Patients considered as cases included children with confirmed sepsis by isolation of a microorganism in blood culture or in a sterile tissue. Patients considered as controls were children with cancer without sepsis and hospitalized children with cardiovascular or general surgery (common conditions in the hospital) with hospital stays at least 72 hours long and no report of sepsis (Figure 2).

The controls offered logistic advantages during enrollment: 1) Controls with cancer and not corroborated sepsis confer two factors: the tumor activity of cancer and the clinical behavior due to chemotherapy, which in many cases can make the clinician suspect that the child is infected, but it is not corroborated by cultures; 2) Surgical cases with no data pointing to infection decrease the risk of misclassifying the child with cancer and suspicion of sepsis, where the gold standard (hemocultures) has 40 to $60 \%$ sensitivity.

\subsection{Strategy to Determine the Patients' Vaccination Status}

It was established by means of a copy of each individual's national vaccination card taken from their file or by asking the social worker or attending physician to fax, scan or email the copy.

\subsection{Operational Definitions}

We considered the following definitions:

1) Not confirmed sepsis [17]: Patient with cancer, hos- pitalized at least during 72 hours, with fever over 38 degrees $\mathrm{C}$ or febricula between 37.5 and 37.9 degrees $\mathrm{C}$ at least twice during 24 hours that deserved sampling of blood, urine and/or cerebrospinal fluid with a negative report with respect to bacterial or mycotic growth.

2) Corroborated nosocomial sepsis [18]: Same as previous one, but with a positive result with respect to a pure strain of a microorganism.

3) Incomplete vaccination [19]: Vaccination schedule lacking the application of at least one of the vaccines contemplated in the institutional card.

\subsection{Sensitivity Analysis}

We took into account some possibilities of bias: We tried to correct the low sensitivity of the blood culture as the patients' classifying datum by including children with some evidence of an active infectious process, such as abscesses in sterile sites. [17] Additionally, we established the percentage of vaccination compliance in both groups (septic vs not septic). Since the vaccination schedules displayed modifications from one year to another, we attempted to decrease the temporality bias by carrying out a matching by age, which ensured that both (cases and controls) were exposed to the same vaccination schedule, the same epidemiologic conditions and the same management at the hospital, including care bynurses and physicians as well as existing standard treatments; all this amounts to exposure to the same known risk factors for the development of nosocomial infections.

A secondary analysis that established the percentage of immunogens administered to the subjects was also car-

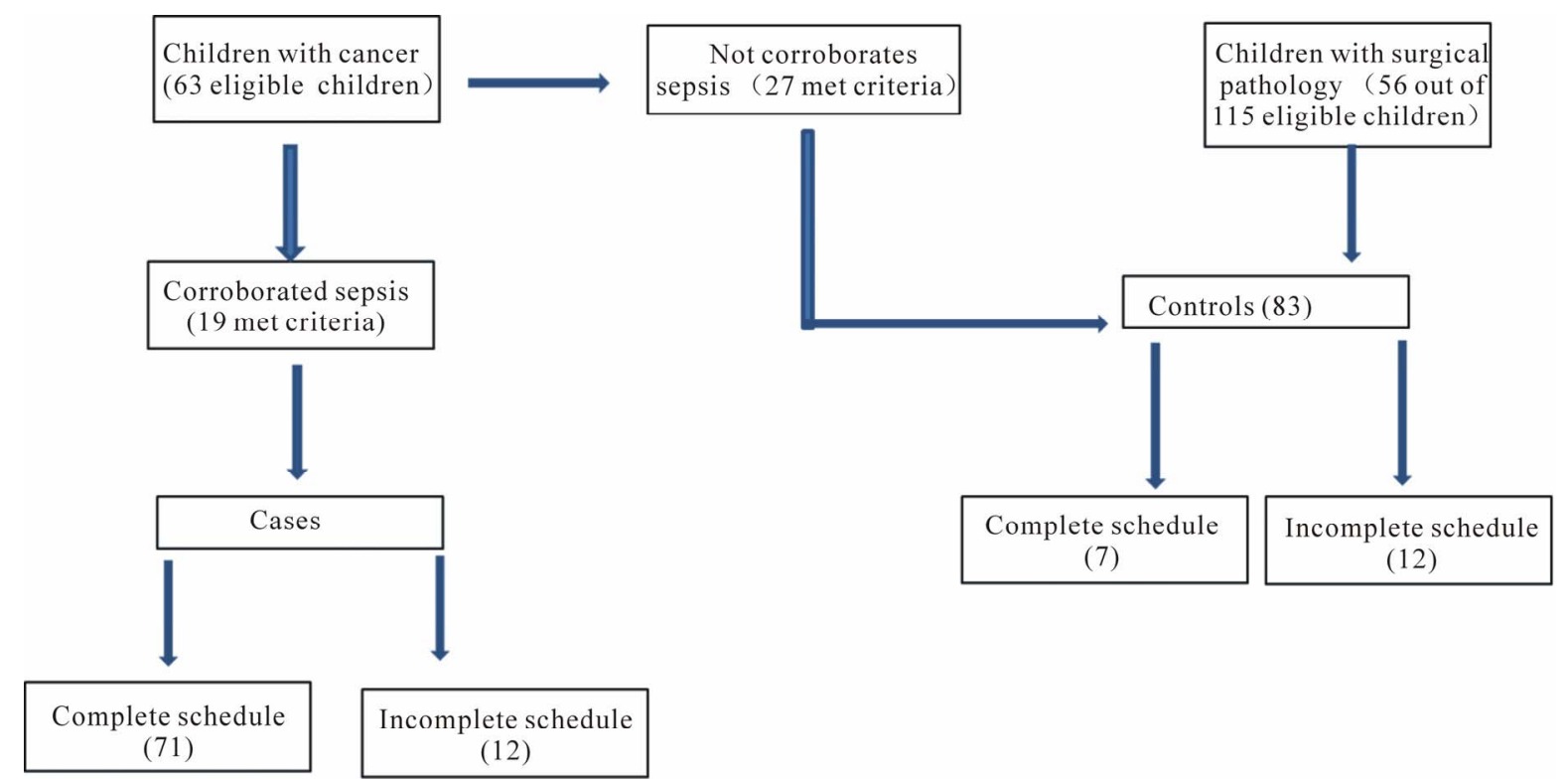

Figure 2. Decision pattern for the inclusion and study of cases and controls. 
ried out.

\subsection{Statistical Analysis}

The categorical variables were compared using a chisquared test, and the normal distribution of the continuous variables by means of a Student's t test. Additionally, bivariate and multivariate logistic regressions were used to determine the factors associated to nosocomial sepsis, and the odds ratio was calculated with confidence intervals of $95 \%$. The percentage of attributable risk was calculated for the variables included in the final model.

Programs EPI Info (version 3.5.3) and SPSS (version 10) were used for the statistical analysis.

\subsection{Ethics}

The study was approved by the institutional research and ethics committee.

\section{Results}

Nineteen children with cancer and confirmed sepsis were included: 15 with hemocultures, two with abscess cultures by aspiration puncture and two with urinary cultures.

Furthermore, 27 children with cancer and negative cultures for microbial growth and 56 hospitalized subjects with cardiovascular or general surgery without data of systemic inflammatory response, due to which no cultures were requested, were included. Vaccination history was confirmed by means of all the patients' vaccination cards.

Out of the 19 cases, $14(73.6 \%)$ had isolation of gramnegative bacilli; three (15.7\%) of gram-positive cocci, and two (10.5\%) were positive for yeast. Median age was six years (range: 10 months-9 years).
Among patients considered as cases, 12 (63\%) had an incomplete vaccination schedule according to their age; eight $(66 \%)$ lacked at least one dose of one of the immunogens recommended in their card, and four (44\%) lacked more than one vaccine. Among the controls, 12 $(14 \%)$ had a complete vaccination schedule.

Through the bivariate analysis, it was found that, besides an incomplete vaccination schedule, the presence of neutropenia and the prolonged use of parenteral nutrition are the factors most strongly associated to nosocomial sepsis. Among them, the highest risk was displayed among patients with an incomplete vaccination schedule: $\mathrm{OR}=$ 10.1 (95\% IC, 3 - 36; $p<0.05$ ) (Table 2). The multivariate analysis was not deemed necessary, due to the minimum quantity of variables with significance, all of them known risk factors for sepsis.

\section{Discussion}

Some studies have addressed the issue of immunization against infectious agents among children with cancer. In this population, the vaccination schedule is interrupted during oncological treatment due to the complications that are likely to arise when live or attenuated microorganisms are applied to an immunocompromised patient $[8,9]$.

The most recent recommendations have been issued by the American Academy of Pediatrics (AAP) and the Centers for Disease Control and Prevention (CDC). Even in this scenario, the protective effectiveness of the vaccines might be affected [20,21].

The association of an incomplete vaccination schedule with specific infectious events, such as nosocomial sepsis, deserves particular considerations:

The immunodeficient status of the host, particularly due to neutropenia,is a factor that is acknowledged as an

Table 2. Bivariate analysis of the variables studied in all of the children included.

\begin{tabular}{|c|c|c|c|c|c|c|}
\hline Variable & Casos $n=19$ & Controls $n=83$ & OR $(95 \% \mathrm{CI})$ & $\mathrm{P}$ & $\mathrm{RR}$ & AP \\
\hline Sex Masculine & 11 & 38 & $1.6(0.5-5)$ & 0.3 & 2.6 & 0.37 \\
\hline Prophylactic antibiotic & 3 & 56 & $0.09(0.02-0.37)$ & 0.00003 & - & - \\
\hline Stay $>14$ days & 18 & 76 & $1.6(0.17-38)$ & 0.6 & 9.7 & 0.37 \\
\hline Neutropenia & 10 & 11 & $7.2(2.1-25)$ & 0.0001 & 1.8 & 0.86 \\
\hline Catheter $>14$ days & 13 & 65 & $0.60(0.18-2)$ & 0.26 & 0.87 & 0.01 \\
\hline Steroids & 15 & 68 & $0.83(0.2-3.4)$ & 0.76 & & \\
\hline TPN $>14$ days & 13 & 29 & $4.0(1.2-13)$ & 0.007 & 1.9 & 0.75 \\
\hline
\end{tabular}

$\mathrm{AP}=$ Attributable Proportion $(\mathrm{OR}-1 / \mathrm{OR}) ; \mathrm{RR}=$ Risk Ratio; $\mathrm{TPN}=$ Total Parenteral Nutrition. 
enhancer for the development of serious infections. Nevertheless, humoral immunity has barely been addressed since its relation seems remote [22-24]. This study constitutes a merely associative — not causal - approach to considering the benefit of applying broad vaccination schedules to prevent diseases that are considered to be serious in a hospital setting. This is particularly so when it is established that the microorganisms causative of nosocomial sepsis are mostly different from those considered in the official vaccination schedules $[25,26]$.

An incomplete vaccination schedule can be considered as a risk factor along with others that have been described as partially responsible for the loss of the opportunity to prevent infections, such as maternal unemployment or the health staff failing to ask about the vaccination schedule $[27,28]$.

Our findings basically suggest that a percentage of the cases of nosocomial infection in our studied populationare strongly associated to an incomplete vaccination schedule prior to the outset of chemotherapy ( $66 \%$ according to the rate of attributable risk). The reasons are similar to those related to factors which are not argued about currently, such as neutropenia.

The study's main limitations include the fact that most likely the studied population should be increased. Moreover, the way in which the information was obtained, by referring to the clinical records, leaves the possibility open for information that cannot be corroborated a posteriori.

Our results point to the fact that although we were dealing with a captive population, there are still cases in which vaccination compliance is low. This condition continues once the oncological diagnosis is established and the vaccines cannot be applied.This issue is far from being the oncologist's or the hematologist's responsibility; rather it should be taken into account by the first contact physicians who are in charge of monitoring the patient's health status, including the vaccination schedule. If this were the case, the percentage of compliance would certainly improve dramatically.

\section{Conclusions}

Approximately from $20 \%$ to $65 \%$ of the cases of serious nosocomial infection could be associated to an incomplete vaccination schedule. Other factors might be added to this, such as the use of catheters and a prolonged hospital stay.

Although the microorganisms found are not precisely the ones that are covered by the vaccination schedule, the association reflects that a good immunological statusboth cellular and humoral - constitutes a factor that could protect against the development of serious infections in immunodepressed children with cancer. Strategies should be designed to improve the vaccination status of the ge- neral pediatric population before children become seriously ill with cancer or with another chronic condition that would preclude the application of vaccines. In accordance with world trends, recommendations made by international organizations on vaccination schedules and immunogen management in cancer patients should be reviewed, and some issues might be adapted to our particular environment.

\section{Acknowledgements}

We are grateful to the National Council of Science and Technology (CONACyT) Mexico, by the supported with the scholarship awarded for postgraduate study; and to Simona Polo of Social work Department from PetroleosMexicanos for her assistance with this investigation

\section{REFERENCES}

[1] R. Wilkinson, "The Impact of Inequality: How to Make Sick Societies Healthier," Preventing Chronic Disease, Vol. 3, No. 1, 2006, p. A26.

[2] A. P. de Leon, M. L. Garcia, M. C. Garcia, F. J. Gómez, J. L. Valdespino, G. Olaiz, R. Rojas, et al., "Tuberculosis and Diabetes in Southern Mexico," Diabetes Care, Vol. 27, No. 7, 2004, pp. 1584-1590. doi:10.2337/diacare.27.7.1584

[3] V. Richardson, J. Hernandez, M. Quintanar, M. Esparza, B. Johnson, C. M. Gomez, U. Parashar and M. Patel, "Effect of Rotavirus Vaccination on Death from Childhood Diarrhea in Mexico," The New England Journal of Medicine, Vol. 362 , No. 4, 2010, pp. 299-305. doi:10.1056/NEJMoa0905211

[4] D. A. Henderson, et al., "Smallpox as a Biological Weapon: Medical and Public Health Management," Journal of the American Medical Association, Vol. 281, No. 22, 1999, pp. 2127-2137.

[5] V. Racaniello, One Hundred Years of Poliovirus Pathogenesis," Virology, Vol. 344, No. 1, 2006, pp. 9-16

[6] M. L. NdeffoMbah, J. Liu, C. T. Bauch, Y. I. Tekel and J. Medlock, "The Impact of Imitation on Vaccination Behavior in Social Contact Networks," PLoS Computational Biology, Vol. 8, No. 4, 2012, Article ID: 1002469. doi:10.1371/journal.pcbi.1002469

[7] F. Feng, D. Rosenbloom, L. Wang and M. Nowak, "Imitation Dynamics of Vaccination Behavior on Social Networks," Proceedings of the Royal Society B: Biological Sciences, Vol. 278, No. 1702, 2011, pp. 42-49.

[8] N. Camille, M. Kotton and M. Poznansky, "Vaccination of Oncology Patients: An Effective Tool and an Opportunity Not to Be Missed," The Oncologist, Vol. 17, No. 1, 2012, pp. 1-2. doi:10.1634/theoncologist.2011-0383

[9] P. Loulergue, O. Mir, J. Alexandre, et al., "Low Influenza Vaccination Rate among Patients Receiving Chemotherapy for Cancer," Annals of Oncology, Vol. 19, No. 9, 2008, p. 1658. doi:10.1093/annonc/mdn531

[10] S. G. Martin, M. D. Mannino, S. Eaton and M. Moss, "The Epidemiology of Sepsis in the United States from 
1979 through 2000," The New England Journal of Medicine, Vol. 348, 2003, pp. 1546-1554. doi:10.1056/NEJMoa022139

[11] A. G.Freifeld and P. Pizzo, "Infectious, Complications in the Pediatric Cancer Patients," In: P. Pizzo and D. Poplack, Eds., Principles and Practice of Pediatric Oncology, JB Lippincott Co., Filadelfia, 1993, pp. 987-992.

[12] V. Y. Dombrovskiy, A. A. Martin, J. Sunderram and H. L. Paz, "Rapid Increase in Hospitalization and Mortality Rates for Severe Sepsis in the United States: A Trend Analysis from 1993 to 2003," Critical Care Medicine, Vol. 35, No. 5, 2007, pp. 1244-1250. doi:10.1097/01.CCM.0000261890.41311.E9

[13] D. C. Angus, W. T. Linde-Zwirble, J. Lidicker, G. Clermont, J. Carcillo and M. R. Pinsky, "Epidemiology of Severe Sepsis in the Unites States: Analysis of Incidence Outcome, and Associated Cost of Care," Critical Care Medicine, Vol. 29, No. 7, 2001, pp. 1303-1310. doi:10.1097/00003246-200107000-00002

[14] F. R. Avila, G. L. Ramírez, A. C. Alpuche, G. J. L. Arredondo and P. J. I. Santos, "Infecciones Nosocomiales en un Hospital Pediátrico," Salud Publica de México, Cuernavaca, 1986.

[15] A. G. Martínez, A. C. Anaya and F. C. Avila, "Incidencia de Bacteriemia y Neumonía Nosocomial en una Unidad de Pediatría," Salud Publica de México, Cuernavaca, 2001. doi:10.1590/S0036-36342001000600001

[16] Instituto Nacional de Estadística y Geografía (INEGI) "Secretaría de Salud/Dirección General de Información en Salud, Base de Datos de Defunciones 2000-2008," INEGI, Aguascalientes, 2010.

[17] B. Goldstein, B. Giroir, A. Randolph, et al., "International Pediatric Sepsis Consensus Conference: Definitions for Sepsis and Organ Dysfunction in Pediatrics," The Pediatric Critical Care Medicine, 2005, Vol. 6, No. 1, 2005, pp. 2-8.

[18] J. S. Garner, W. R. Jarvis, T. G. Emori, T. C. Horan and J. M. Hughes, "CDC Definitions for Nosocomial Infections. In: R. N. Olmsted, ed., APIC Infection Control and Applied Epidemiology: Principles and Practice, Mosby, St. Louis, 1996, pp. A1-A20.

[19] J. V. Jani, C. De Schacht1, I. V Jani and G. Bjune, "Risk factors for Incomplete Vaccination and Missed Opportunity for Immunization in Rural Mozambique," BMC Pub- lic Health, 2008, Vol. 8, No. 2, p. 161. doi:10.1186/1471-2458-8-161

[20] Centers for Disease Control and Prevention, "Prevención y Control de la influenza con Vacunas: Recomendaciones del Comité Asesor Sobre Prácticas de Inmunización (ACIP) 2011," 2011 Morbidity and Mortality Weekly Report (MMWR), Druid Hills, 2011.

[21] Centers for Disease Control and Prevention, "Prevención y Control de la Influenza con Vacunas: Recomendaciones del Comité Asesor Sobre Prácticas de Inmunización (ACIP) 2010," 2010 Morbidity and Mortality Weekly Report (MMWR), Druid Hills, 2010.

[22] G. L. G. Montalván, "Sepsis Severa y Shock Séptico en el Niño. Aún no Todo Está Dicho," Pan American Journal of Public Health, Vol. 26, No. 6, 2009, pp. 562-564.

[23] F. J. Reyna, L. C. V. Richardson and V. P. Vidal "De las Definiciones, las Vacunas y la Identificación del Paciente Séptico en Pediatría," Pan American Journal of Public Health, Vol. 27, No. 6, 2010, pp. 469-470. doi:10.1590/S1020-49892010000600010

[24] T. Lehrnbecher, C. Foster, N. Vázquez, C. L. Mackall and S. J. Chanock, "Therapy-Induced Alterations in Host Defense in Children Receiving Therapy for Cancer," Journal of Pediatric Hematology/Oncology, Vol. 19, No. 5, 1997, pp. 399-417. doi:10.1097/00043426-199709000-00001

[25] P. A. Pizzo, "Management of Fever in Patients with Cancer and Treatment-Induced Neutropenia," The New England Journal of Medicine 1993; Vol. 328, No. 18, pp. 1323-1332. doi:10.1056/NEJM199305063281808

[26] R. N. Jones, “Contemporary Antimicronial Susceptibility Pattems of Bacterial Pathogens Commonly Assoclated with Febrile Patients with Neutropenia," Clinical Infectious Diseases, Vol. 29, No. 3, 1999, pp. 495-502. doi:10.1086/598621

[27] M. Macias, G. A. Jarquin, P. Gutierrez, M. A. Rodriguez, N. Gonzalez and P. Saltigeral, "Factores de Riesgo para Esquemas de Vacunación Incompletos en Niños de 6 a 60 Meses en el Instituto Nacional de Pediatría," Revista de Enfermedades Infecciosas en Pediatría, Vol. 22, No. 86, 2008, pp. 41-47.

[28] E. Holt, B. Guyer, N. Hughart, et al., "The Contribution of Missed Opportunities to Childhood Immunization in Baltimore," Pediatrics, Vol. 97, No. 4, 1996, pp. 474480. 